\title{
LA BASE DE DATOS DE RESOLUCIONES DE CONSULTAS TRIBUTARIAS COMO HERRAMIENTA DE INNOVACIÓN DOCENTE ${ }^{1}$
}

\section{The database of tax reference resolutions as a teaching innovation tool}

\section{RESUMEN}

El presente artículo versa sobre la utilización como instrumento de innovación docente de la base de datos de resoluciones de consultas vinculantes de la Dirección General de Tributos, de fácil accesibilidad por su desarrollo informatizado on line y con un poderoso motor de búsqueda basado en el software de gestión de bibliotecas Knosys. El objetivo último será determinar la actitud del alumnado frente a la utilización de la base de datos en las clases. El estudio se divide en tres bloques: el primero, dedicado al régimen jurídico de las consultas tributarias; otro gran apartado, relativo a la utilización de la herramienta por parte de los alumnos o profesionales en general; y un último apartado, donde se centra el objeto de estudio, dedicado al valor que puede tener esta herramienta de cara a la mejora de la enseñanza del Derecho financiero y tributario.

\section{PALABRAS CLAVES}

Derecho tributario, base de datos, e-government, innovación educativa.

\begin{abstract}
This paper deals with the use as a teaching innovation tool of the database of collaboration queries of the General Directorate of Taxes, of easy accessibility for its computerized development online and with a powerful search engine based on the management software of Knosys libraries. The ultimate goal will be to determine the attitude of students against the use of the database in the classes. The study is divided into three blocks: the first, dedicated to the legal regime of tax consultations; another large section, relating to the use of the tool by students or professionals in general; and a last section, where the object of study is focused, dedicated to the value that this tool can have in order to improve the teaching of financial and tax law.
\end{abstract}

\section{KEYWORDS}

Tax Law, database, e-government, educational innovation.

\footnotetext{
1 Este artículo se enmarca dentro los materiales generados en la estancia de investigación realizada en la Universidad de Málaga, en la línea de investigación de derecho de la informática.
} 
Sumario: 1. Consideraciones previas. 2. Aproximación al régimen jurídico de las consultas tributarias de la Dirección General de Tributos. 2.1. Delimitación conceptual, caracteres y efectos. 2.2. La presentación de las consultas tributarias. 3. La base de datos de consultas tributarias. 3.1. Introducción a la base de datos Knosys de la Dirección General de Tributos. 3.2. Evolución de la base de datos. 3.3. Los operadores de la base de datos. 4. La utilización como herramienta de innovación docente de la base de datos de consultas tributarias. 5. Consideraciones finales. Bibliografía.

\section{Consideraciones previas.}

La Dirección General de Tributos (DGT, en lo sucesivo), en desarrollo del artículo 85.2 de la Ley 58/2003, de 17 de diciembre, General Tributaria (en adelante, LGT), que señala que las actividades de información y asistencia de la administración tributaria se instrumentarán, entre otras formas, a través de las contestaciones a consultas escritas, elaboró unos compendios de las referidas resoluciones, que con el tiempo, por su grosor, fueron denominados cariñosamente como los libros gordos de «petete». Para agilizar la publicación y el tratamiento de estos datos, la información se informatizó, creando dicha Dirección General, una base de datos de carácter pionero e innovador.

En la actualidad dicha base de datos se encuentra en línea en el portal del Ministerio de Hacienda, y tiene un indudable valor docente porque sistematiza valiosa información que ayuda al alumno a conocer el criterio de la Administración respecto de ciertos aspectos de la aplicación de los tributos que pueden resultar dudosos.

La base de datos de resoluciones de consultas vinculantes de la DGT se configura en un verdadero «digesto» del ámbito tributario, de fácil accesibilidad por su desarrollo informatizado on line y con un poderoso motor de búsqueda basado en el software de gestión de bibliotecas Knosys.

Los objetivos del presente estudio comprenden la delimitación de la figura de la resolución a las consultas vinculantes, el procedimiento telemático de presentación de consultas, el análisis de la base de datos y su evolución en el tiempo y el estudio de los comandos para operar con ella y, por último, de la recepción por el alumnado como resultado de su utilización en clases prácticas la asignatura fiscalidad empresarial.

\section{Aproximación al régimen jurídico de las consultas tributarias de la Dirección General de Tributos.}

El objeto de estudio de este artículo es la base de datos de resoluciones de consultas tributarias, pero a modo de somera introducción, se incluye el presente epígrafe con la estructura básica de esta institución jurídica, así como del trámite de presentación.

\subsection{Delimitación conceptual, caracteres y efectos.}

Define VILLAVERDE GÓMEZ la consulta tributaria como: «el derecho de cualquier obligado tributario a solicitar por escrito a la Administración tributaria competente que ésta le informe, también por escrito, acerca de cuál es la interpretación administrativa de 
las normas tributarias aplicables a un caso concreto que le afecta, quedando la Administración actuante vinculada respecto de la contestación facilitada» ${ }^{2}$.

Como expresión legal del concepto, los artículos 88 y 89 LGT y su desarrollo reglamentario en los artículos 65, 66, 67 y 68 del Real Decreto 1.065/2.007, de 27 de julio, por el que se aprueba el Reglamento General de las actuaciones y los procedimientos de gestión e inspección tributaria y de desarrollo de las normas comunes de los procedimientos de aplicación de los tributos (RGGI, en adelante), señalan que los obligados tributarios podrán formular a la Administración tributaria consultas para conocer el criterio de la misma respecto de la aplicación de ciertos aspectos de las normas que pueden suscitar equívocos $\mathrm{y}$, en general, respecto del régimen, clasificación o calificación tributaria que en cada caso les corresponda.

Es posible colegir los caracteres de las citadas consultas tributarias extrayéndolos del concepto antes reseñado ${ }^{3}$.

En primer lugar, el artículo 88 LGT establece que las consultas tributarias pueden ser planteadas por cualquier obligado tributario con un interés legítimo, e incluso por determinados colectivos legitimados. Así, podrán formular consultas tributarias los colegios profesionales, cámaras de comercio, patronales, sindicatos, asociaciones de consumidores, asociaciones o fundaciones en interés de personas con discapacidad, asociaciones empresariales y colegios profesionales. También las federaciones que agrupen a los referidos entes, cuando la materia tributaria tratada afecte a la generalidad de sus miembros.

Asimismo, pueden realizar consultas los potenciales obligados tributarios, previamente a que se produzca el presupuesto de hecho y siendo previsible que acontezca, tal y como se señala en el artículo 66.1.b) RGGI ${ }^{4}$.

Por otra parte, su resolución compete a los órganos de la Administración tributaria que tengan atribuida la potestad interpretativa ${ }^{5}$. Y, territorialmente, se han establecido unas reglas de distribución competencial entre las Comunidades Autónomas y el Estado, cuando nos hallemos ante tributos cedidos ${ }^{6}$. Por tanto, la competencia de resolución no solo pertenece a un único órgano. Esta asignación es respetuosa con el carácter de legislación básica del artículo 88.5 LGT, consecuencia de la competencia para el estudio,

\footnotetext{
${ }^{2}$ VILLAVERDE GÓMEZ, M. B., "Un supuesto de interpretación de las normas tributarias: los efectos jurídicos de las contestaciones a consultas", Dereito: Revista xuridica da Universidade de Santiago de Compostela, n. 1, 2013, p. 687.

${ }^{3}$ Seguimos, para la enumeración de caracteres, la clasificación realizada por ADAME MARTÍNEZ, F. D., en "Naturaleza jurídica y efectos de las contestaciones a consultas tributarias", Crónica tributaria, n. 119, 2006, p. 10 y VILLAVERDE GÓMEZ, M. B., en op. cit., p. 688.

${ }^{4}$ El meritado artículo indica que el escrito de iniciación del procedimiento de consulta debe incluir la «manifestación expresa de si en el momento de presentar el escrito se está tramitando o no un procedimiento, recurso o reclamación económico-administrativa relacionado con el régimen, clasificación o calificación tributaria que le corresponda planteado en la consulta, salvo que esta sea formulada por las entidades a las que se refiere el artículo 88.3 de la LGT».

${ }^{5}$ Véase el artículo 88.5 LGT.

${ }^{6}$ El artículo 55.2.a) de la Ley 22/2009, de 18 de diciembre, por la que se regula el sistema de financiación de las Comunidades Autónomas de régimen común y Ciudades con Estatuto de Autonomía y se modifican determinadas normas tributarias. Indica este artículo 55.2. que «No son objeto de delegación las siguientes competencias: a) La contestación de las consultas reguladas en los artículos 88 y 89 de la LGT, salvo en lo que se refiera a la aplicación de las disposiciones dictadas por la Comunidad Autónoma en el ejercicio de sus competencias».
} 
elaboración y propuesta por parte de las Comunidades Autónomas, respecto al sistema de financiación ${ }^{7}$.

En referencia a sus efectos, serán vinculantes para los órganos de la Administración tributaria encargados de la aplicación de los tributos en el presupuesto de hecho del consultante. Dicha vinculación se establece únicamente para las Administraciones. Los obligados tributarios tienen la libertad de aplicar el criterio contenido en la resolución o cualquiera que consideren más fundado ${ }^{8}$.

La LGT de 1963 diferenciaba las consultas vinculantes y consultas no vinculantes. Así, las consultas se calificaban según si producían efectos vinculantes o no. A las vinculantes se las calificaba como actos administrativos; sin embargo, a las no vinculantes se les revestía de un carácter meramente informativo. En la actualidad, este debate es trascendente porque, desde la entrada en vigor de la «nueva» LGT, regulada por la Ley $58 / 2003$, se les atribuyen efectos vinculantes a todas las resoluciones a consultas tributarias, si cumplen los requisitos legales. Se mantienen aquellas que la antigua LGT denominaba «consultas generales» en un apartado de la base de datos ${ }^{9}$.

Con respecto a la extensión de efectos de una contestación a otros obligados tributarios, la LGT extiende el efecto vinculante a cualquier obligado, siempre que exista identidad entre los hechos y circunstancias de dicho obligado y los que se incluyan en la contestación a la consulta ${ }^{10}$.

Debemos, para subrayar esta extensión de efectos a terceros, mencionar la conexión con la previsión del artículo 179 LGT en su apartado 2. d) ${ }^{11}$.

Asimismo, el artículo 179.2. LGT, señala: «Las acciones u omisiones tipificadas en las leyes no darán lugar a responsabilidad por infracción tributaria en los siguientes supuestos: (...) d)... si el obligado tributario ajusta su actuación a los criterios manifestados por la Administración en la contestación a una consulta formulada por otro obligado, siempre que entre sus circunstancias y las mencionadas en la contestación a la consulta exista una igualdad sustancial que permita entender aplicables dichos criterios y éstos no hayan sido modificados». Para VILLAVERDE GÓMEZ esta exoneración de responsabilidad no actúa únicamente en el plano sancionador tributario, sino que es posible la extensión al ámbito penal de responsabilidad de las actuaciones sobre la base de una consulta tributaria, porque derivaría una ausencia de culpabilidad, por haber incurrido en error invencible de prohibición ${ }^{12}$.

\footnotetext{
${ }^{7}$ CHAMS EL DIN DELGADO, J. A., "Información y asistencia a los obligados tributarios: Las consultas vinculantes", Hacienda Canaria, n. 10, 2004, p. 180.

${ }^{8}$ MARTÍNEZ GINER, L. A., "Las consultas vinculantes en el marco de una inspección tributaria: una propuesta sobre la base de la solución del derecho alemán", Revista de información fiscal, n. 61, 2004, p. 28.

${ }^{9}$ FALCÓN Y TELLA, R., nos relata este cambio en "El carácter vinculante de la doctrina sentada al resolver consultas tributarias y reclamaciones económico-administrativas", Quincena fiscal, n. 19, 2004, pp. 5-8.

${ }^{10}$ A este respecto, en el estudio de qué se entiende por «identidad entre los hechos y circunstancias», se puede abundar en el artículo de FERNÁNDEZ PAVÉS, M. J., "La nueva regulación aplicable a las consultas con contestación vinculante", Revista de información fiscal, n. 24, 1997, pp. 7-30.

${ }^{11}$ MENÉNDEZ MORENO, A., "De la anécdota a la categoría: (a propósito de las consultas vinculantes y del régimen de estimación objetiva)", Quincena fiscal, n. 12, 2014, p. 12.

${ }^{12}$ VILLAVERDE GÓMEZ, M. B., op. cit., p. 700.
} 
Por último, indicar que las contestaciones no son recurribles, por expreso mandato del artículo 89.4 LGT, aunque sí lo son a través de los actos de aplicación que recojan sus criterios interpretativos.

\subsection{La presentación de las consultas tributarias.}

Las consultas tributarias escritas se formularán -artículo 88.2 LGT-, mediante escrito dirigido al órgano competente para su contestación, la DGT. En el referido escrito deberá identificarse al consultante, explicar el caso y concretar la cuestión planteada. Asimismo, se acompañará la documentación necesaria ${ }^{13}$.

Lo usual en la actualidad es la presentación de la consulta rellenando un formulario electrónico en la página web de la DGT en el portal del Ministerio de Hacienda. Existe, además, la obligación acompañar el formulario de datos complementarios específicos en formato PDF. La presentación en el registro electrónico será conforme a lo que se establece en el artículo 16.4.a) de la Ley 39/2015, es decir, se realizará en el registro electrónico de la Administración u Organismo al que se dirijan, así como en los restantes registros electrónicos del Estado, Comunidades Autónomas, entidades que integran la Administración Local y el sector público institucional.

La Administración tributaria competente deberá contestar por escrito las consultas en el plazo de seis meses desde su presentación. La falta de contestación en dicho plazo no implicará la aceptación de los criterios expresados en el escrito de la consulta. Por propia experiencia se puede afirmar que hay tendencia a agotar el plazo.

La presentación y contestación de las consultas no interrumpirá los plazos establecidos en las normas tributarias para el cumplimiento de las obligaciones tributarias -artículo 89.3 LGT-.

\section{La base de datos de consultas tributarias.}

El presente epígrafe es un estudio de la parte técnica de la base de datos de consultas tributarias, que incluye su evolución y su manera de operar.

\subsection{Introducción a la base de datos Knosys de la Dirección General de Tributos.}

Como hemos señalado en el epígrafe de cuestiones previas, la compilación de resoluciones de consultas tributarias se hizo muy voluminosa $\mathrm{y}$, por tanto, casi inmanejable -de ahí su apodo de «petete»-. Para dar solución a este problema, a mediados de la década de los noventa, se utilizaron medios informáticos. Por aquel entonces, Knosys -un sistema de gestión documental- era, con mucha diferencia, el software más extendido en las bibliotecas de nuestro país ${ }^{14}$. Se trata de un programa de gestión de base de datos que puede manejar registros textuales con información estructurada mediante campos. Dado que la extensión de tales campos es variable, también es posible utilizarlo como sistema de gestión de documentos de texto completo, ya sea importando

\footnotetext{
13 CHECA GONZÁLEZ señala que si no se aporta información, como ocurre en muchos casos, la DGT incluye verdaderas cláusulas de estilo en las cuales manifiesta que el criterio se configura conforme a los hechos y documentos aportados. CHECA GONZÁLEZ, C., "Acerca del procedimiento administrativo de elaboración de las normas tributarias. Aportaciones de los órganos consultivos sobre técnica normativa", Documentos - Instituto de Estudios Fiscales, n. 30, 2009, p. 262.

${ }^{14}$ Sobre los albores de Knosys se ha consultado, BOTELLA BONILLA, E.; LUENGO MARQUINA, J. C.; ALVAREZ, M. I., Knosys, aplicaciones de bases de datos documentales en la enseñanza, Ministerio de Educación y Ciencia, 1990.
} 
directamente los documentos o creándolos con su propio editor ${ }^{15}$. A todo esto, se unió, posteriormente, la capacidad de publicar una interfaz web para su acceso on line.

\subsection{Evolución de la base de datos.}

Podemos dividir la evolución de la base de datos en tres etapas: una primera, en la que el programa de gestión y la base de datos, se distribuían en $\mathrm{CD}$; una segunda, que abarca desde principios del dos mil hasta el verano del año 2015, en la que el acceso se produce on line; y una tercera, en la que estamos, en la que se modifica el software, volcando los registros a una base de datos más potente y utilizando páginas dinámicas de última generación en la interfaz web.

Es preciso desarrollar lo que ocurrió en este punto, porque la modificación de la operativa on line, ocurrida en 2015, tenía sus beneficios, pero también sus inconvenientes.

En ambas se parte de la página web del Ministerio de Hacienda, que nos posibilita la presentación telemática de una consulta que queramos realizar y se nos enlaza a la normativa de referencia. También se nos muestran los enlaces a la Base de Datos de Consultas, tanto generales, como vinculantes.

Si accedemos a la Base de Datos, nos encontramos con un formulario con los siguientes campos: NUM-CONSULTA, FECHA-SALIDA, NORMATIVA, CUESTION-PLANTEADA, DESCRIPCION-HECHOS, TEXTO LIBRE.

Hasta 2015 se podía realizar la búsqueda utilizando el mismo motor de búsqueda de Knosys, que es robusto, pero, por entonces, la implementación web no era demasiado buena y el motor de búsqueda no era muy flexible. Por ejemplo, si hacíamos una búsqueda rápida, de consultas relacionadas con «impuesto sobre sucesiones excesos de adjudicación», utilizando el campo TEXTO LIBRE o DESCRIPCION-HECHOS. El resultado era: «no se encontraron documentos». Lo cual era bastante descorazonador. Era imposible que no existieran consultas vinculantes con esos términos, convendréis conmigo. El motor de búsqueda de Knosys no encontraba búsquedas tan abiertas, con lo que había que filtrar más la búsqueda y perderíamos un tiempo precioso.

Lo bueno es que hasta 2015, el sistema Knosys no funcionaba completando las variables incrustadas en el HTML dinámico, sino que se generaban las páginas consultas individuales en HTML plano a las que se accedía mediante una página de enlaces dinámica. ¿Qué significa esto? Que podíamos utilizar para realizar las búsquedas el mejor motor de búsquedas, Google, dado que las páginas de consultas eran mero texto HTML, que se indexaban por los bots de Google.

Así, hasta 2015, para hacer las búsquedas en la base de datos de consultas vinculantes se podía utilizar Google, con el comando «site:» para apuntar a la web y se encontraban resultados que el software propietario de Knosys no encontraba. De igual modo, se podían utilizar otros comandos, además, como el filtrado de resultados del último mes o del último año, que resultan muy cómodos para las búsquedas.

\footnotetext{
${ }^{15}$ La potencia del editor combinada con la de la base de datos fue una razón de peso para su adopción, como se señala en la introducción del libro FERNÁNDEZ CORRAL, B.; MARTÍNEZ HERNÁNDEZ, M., Base de datos Knosys, Bilbao: Tekel, D.L., 1992.
} 

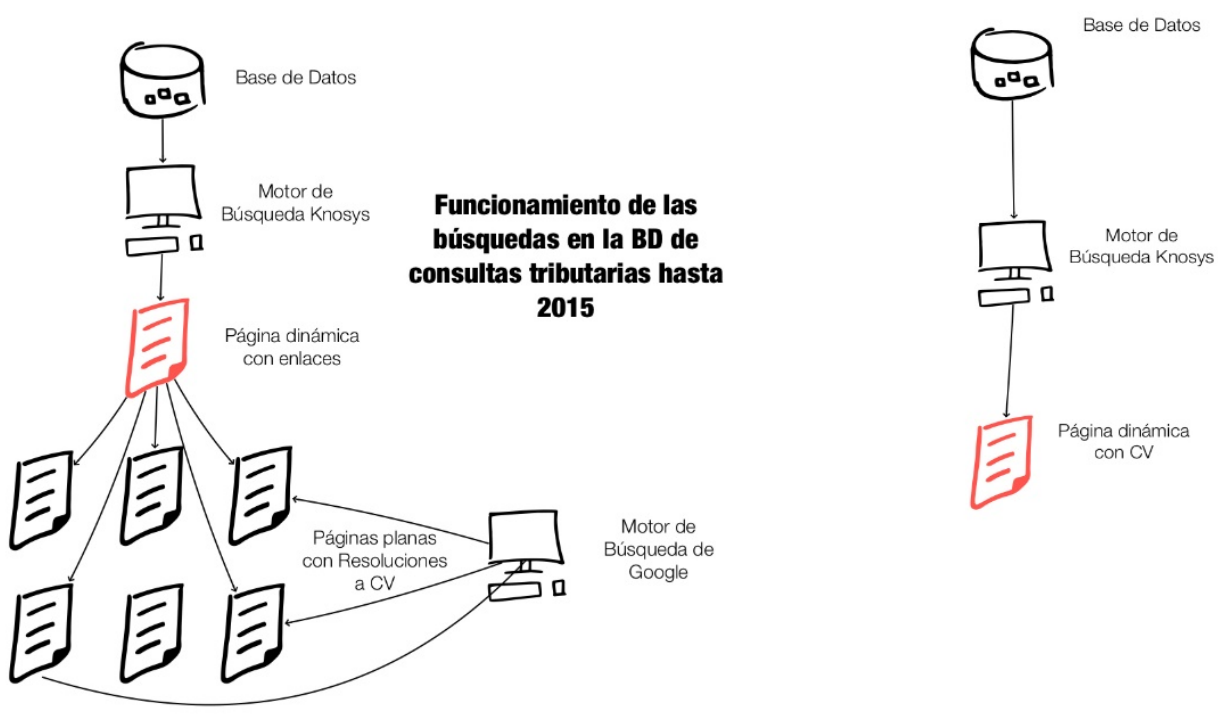

Funcionamiento de las búsquedas en la BD de consultas tributarias desde 2016

Como se puede observar las operativas son diferentes, no es posible utilizar un motor de búsqueda externo, lo cual restringe posibilidades; ahora bien, es posible, que el coste de mantenimiento sea menor y la organización superior, al no tener generada tanta página en texto plano, sino solamente una dinámica que se modifica por búsqueda.

\subsection{Los operadores de la base de datos.}

Centrándonos en el motor de búsqueda de Knosys -que como hemos visto es la única opción actual-, se nos presenta la posibilidad de realizar búsquedas más certeras con el aprendizaje de ciertas técnicas, operadores y comandos.

El software de gestión Knosys siempre destacó por tener un lenguaje de búsqueda (query) muy potente, que permite realizar búsquedas utilizando palabras o frases, sin necesidad de comandos que entorpezcan la operatividad para los no informáticos. Además, las palabras o las frases se pueden combinar mediante los tres operadores booleanos clásicos (AND, OR, NOT en su versión de programación, Y, O y .NO). Y soporta operadores de proximidad y delimitadores, que pueden ser de gran utilidad a la hora de interrogar a la base de datos ${ }^{16}$.

Knosys también acepta truncamientos $(*)$ para que la base de datos te devuelva todos los posibles finales de una expresión. Por ejemplo, utilizando como pregunta $<$ contrib*>, $^{*}$, el sistema busca documentos que tengan las palabras "contribución", "contribuyente", "contribuyentes", etc. Admite, por otra parte, la utilización de máscaras (?) en sustitución de uno o más caracteres en los términos de búsqueda ${ }^{17}$.

Finalmente, el uso de comillas permite encontrar un grupo de palabras de forma literal y el uso de paréntesis permiten combinar todas las opciones indicadas en una única búsqueda.

\footnotetext{
${ }^{16}$ FERNÁNDEZ CORRAL, B.; MARTÍNEZ HERNÁNDEZ, M., Base de datos Knosys, op. cit., p.20.

${ }^{17}$ BOTELLA BONILLA, E.; LUENGO MARQUINA, J. C.; ALVAREZ, M. I., Knosys, aplicaciones de bases de datos documentales en la enseñanza, op. cit., p. 45.
} 


\section{La utilización como herramienta de innovación docente de la base de datos de consultas tributarias.}

\subsection{Modo de inserción en clase del uso de la base de datos de consultas tributarias.}

La investigación-exploración como metodología docente aprovechando las bases de datos de libre acceso no es una novedad ${ }^{18}$. Sin embargo, su adaptación a algo tan concreto como la docencia del Derecho Financiero y Tributario, no es una práctica muy extendida.

En primer lugar, se da una clase magistral sobre el régimen jurídico de las consultas, al modo que se ha realizado en el primer epígrafe de este artículo. Seguidamente, se aborda técnicamente el funcionamiento de la base de datos y se realizan búsquedas ejemplificativas por campos concretos. Posteriormente, nos centramos en una cuestión específica y se pide al alumno que explore la base en busca de resoluciones sobre la materia, la misma debe aprovecharse para explicar conceptos complejos que pueden aparecer o que puedan ser relevantes para su compresión, así como para responder a las preguntas que se planteen. Respecto a esto último, el docente, puede ir llamando la atención sobre cuestiones que luego serán debatidas o que considere relevantes para el aprendizaje.

Resulta estimulante, para docente y alumnos, plantear el método inductivo y partir de lo concreto a lo general, como el deductivo, que supone el planteamiento opuesto. Así, se plantean actividades en las que, partiendo de casos concretos conocemos el criterio de la DGT sobre la norma; y, en otras ocasiones, partimos del estudio de una norma que nos interesa y, a través de la base de datos, conocemos las situaciones de hecho en las que se ha aplicado.

\subsection{Metodología de la investigación.}

Por último, ciñéndonos al objetivo de este estudio, que es determinar la actitud del alumnado frente a la utilización de la base de datos de consultas vinculantes en clase hemos realizado un análisis en el que se ha utilizado una metodología descriptiva, mediante la elaboración una escala tipo Likert, en cuestionario, dirigido al alumnado, con cinco opciones que van desde «totalmente de acuerdo», «de acuerdo», «no puedo opinar», «desacuerdo» y «totalmente en desacuerdo». El cuestionario se compone de 4 ítems estructurados en cuatro categorías relativas a la importancia, satisfacción, interés y dificultad de la utilización de la base de datos de consultas tributarias (Tabla 1).

\footnotetext{
${ }^{18}$ A mayor abundamiento, los siguientes artículos PAVÓN PÉREZ, J. A., "El derecho administrativo a través de la consulta de bases de datos jurídicas accesibles en red y la utilización de redes sociales (blogs jurídicos)", en CAMISÓN YAGÜE, J. A. (Dir.), Experiencias de Innovación docente en los Estudios Jurídicos: una visión práctica., [Sevilla] : Grupo de Investigación «Fiscalitas \& Iuris» de la Universidad de Extremadura, 2016, pp. 130-136.

PÉREZ CARRILLO, E. F., "Bases de datos electrónicas como apoyo a la docencia y a la coordinación de contenidos impartidos en los estudios jurídicos: el ejemplo de Eur Lex", en ECHEBARRÍA SÁENZ, M. (Dir.), Coordinación y planificación en los estudios de Derecho [Recurso electrónico]: 15 al 16 de septiembre 2012 : Universidad de Valladolid, Valladolid : Universidad de Valladolid, 2012, pp. 142-171; PEREZ DEL HOYO, R.; ANDÚJAR-MONTOYA, M. D.; SENTANA GADEA, I.; GONZÁLEZ AVILÉS, Á. B.; GARCÍA GONZÁLEZ, E., "Las bases de datos de acceso libre como metodología docente", en Memorias del Programa de Redes-I3CE de calidad, innovación e investigación en docencia universitaria: Convocatoria 2017-18, Alicante: Universidad de Alicante, Instituto de Ciencias de la Educación (ICE), 2018, pp. 2073-2079.
} 


\begin{tabular}{|l|c|}
\hline Faceta & n \\
\hline Importancia & 1 \\
\hline Interés & 2 \\
\hline Dificultad & 3 \\
\hline Satisfacción & 4 \\
\hline
\end{tabular}

Tabla 1. Distribución de Cuestiones

La muestra estuvo formada por 91 estudiantes de Derecho Financiero y Tributario; 63 son de sexo femenino y 28 son de sexo masculino; la edad promedio se sitúa en 21,19 años, el 50\% de los alumnos encuestados tienen 21 años, la edad más común fue de 20 años. 51 alumnos eran del turno de la mañana, 40 del turno de la tarde.

Los datos obtenidos fueron analizados mediante el software SPSS 15.0. y se presentaron los siguientes resultados en las facetas de: importancia, satisfacción, interés y dificultad. Mostrando las tablas de frecuencias y porcentajes para cada uno, con sus respetivas interpretaciones (tabla 2).

\begin{tabular}{|c|c|c|c|c|c|c|c|c|c|c|}
\hline \multirow{2}{*}{ Ítems } & \multicolumn{2}{|c|}{5} & \multicolumn{2}{|c|}{4} & \multicolumn{2}{|r|}{3} & \multicolumn{2}{|c|}{2} & \multicolumn{2}{|c|}{1} \\
\hline & $\mathrm{F}$ & $\%$ & $\mathrm{f}$ & $\%$ & $\mathrm{f}$ & $\%$ & $\mathrm{f}$ & $\%$ & $\mathrm{f}$ & $\%$ \\
\hline 1.Es útil la $\mathrm{BD}$ de consultas tributarias & 75 & 82,4 & 16 & 17,6 & 0 & 0 & 0 & 0 & 0 & 0 \\
\hline $\begin{array}{l}\text { 2.Me resulta indiferente la BD de consultas } \\
\text { tributarias }\end{array}$ & 1 & 1,1 & 3 & 3,3 & 5 & 5,5 & 42 & $\mid 46,2$ & 40 & 44 \\
\hline 3.La BD me resulta difícil de utilizar & 1 & 1,1 & 0 & 0 & |14 & 15,4 & 36 & $|39,5|$ & 40 & 44 \\
\hline 4.Me gusta utilizar la $\mathrm{BD}$ & 30 & 33 & 40 & 44 & 18 & 19,8 & 3 & 3.3 & 0 & 0 \\
\hline
\end{tabular}

Tabla 2. Distribución de respuesta de los estudiantes al cuestionario-escala

A continuación, destacamos los porcentajes más relevantes de la tabla. En la categoría importancia, item referido a la utilidad del paquete estadístico para procesar datos, se observa que un $82,4 \%$ está muy de acuerdo. En el interés demostrado, el 46,2\% muestra su desacuerdo respecto al elemento 2, en la afirmación de que me es indiferente. Con respecto a la dificultad de utilización el 15,4\% del alumnado está de acuerdo, con lo que pocos alumnos lo consideraron difícil. Al considerar la categoría satisfacción, ítem 4, se observa en la opción de acuerdo un porcentaje de $44 \%$ por lo que el estudiante se siente bien durante la clase con el uso de la base de datos.

\section{Consideraciones finales.}

Primera.- La utilidad de las resoluciones a consultas vinculantes por la DGT está fuera de dudas en la práctica de la abogacía y asesoría tributaria. Su régimen jurídico es, como hemos señalado, apropiado, delimitando la figura y, a partir de una idea clásica un digesto-, ha configurado un sistema dinámico.

Segunda.- La informatización del compendio en una base de datos ha supuesto un mejor acceso y estudio de la misma. El volumen de la información lo requería, de ahí que el volcado de datos del justamente llamado libro de "petete", fuera tan necesario.

Tercera.- La base de datos ha evolucionado conforme a los tiempos. Primero, en ordenadores centrales de acceso en la propia DGT, después en su formato CD y DVD; y en su última fase, on line. 
Cuarta.- Incluso en su última fase de desarrollo en la web se han producido modificaciones, como la que se operó en 2015 , en la que se eliminaron las consultas completas en HTML plano, por una página de resultado final que carga la consulta en variables. Lo que ha supuesto un servidor más ordenado, pero ha eliminado la posibilidad de utilizar motores de búsqueda externos.

Quinta.- La utilización de la base de datos de resoluciones tributarias como herramienta de innovación docente resulta sumamente interesante al mostrar el criterio de la Administración tributaria en supuestos de hecho reales. Los resultados de la encuesta desarrollada por el autor corroboran la buena predisposición del alumnado hacia la utilización de la base de datos objeto de observación como herramienta de soporte docente.

\section{Bibliografía.}

- ADAME MARTÍNEZ, F. D., "Naturaleza jurídica y efectos de las contestaciones a consultas tributarias", Crónica tributaria, n 119, 2006, pp. 9-44.

- ADAME MARTÍNEZ, F. D., "Régimen Jurídico de las consultas tributarias en derecho español y comparado", Documentos - Instituto de Estudios Fiscales, $\mathrm{n}^{\mathrm{o}}$ 21, 2001, pp. 1-44.

- BOTELlA BONILlA, E.; LUENGO MARQUINA, J. C.; ALVAREZ, M. I., Knosys, aplicaciones de bases de datos documentales en la enseñanza, Ministerio de Educación y Ciencia, 1990.

- CHAMS EL DIN DELGADO, J. A., "Información y asistencia a los obligados tributarios: Las consultas vinculantes", Hacienda Canaria, no 10, 2004, pp. 175-191.

- CHECA GONZÁLEZ, C., "Acerca del procedimiento administrativo de elaboración de las normas tributarias. Aportaciones de los órganos consultivos sobre técnica normativa", Documentos - Instituto de Estudios Fiscales, n 30, 2009, pp. 261-274.

- FALCÓN Y TELLA, R., "El carácter vinculante de la doctrina sentada al resolver consultas tributarias y reclamaciones económico-administrativas", Quincena fiscal, $\mathrm{n}^{\mathrm{o}} 19,2004$, pp. 5-8.

-FERNÁNDEZ CORRAL, B.; MARTÍNEZ HERNÁNDEZ, M., Base de datos Knosys, Bilbao: Tekel, D.L. 1992.

- FERNÁNDEZ PAVÉS, M. J., "La nueva regulación aplicable a las consultas con contestación vinculante", Revista de información fiscal, n 24, 1997, pp. 7-30.

- MARTÍNEZ GINER, L. A., "Las consultas vinculantes en el marco de una inspección tributaria: una propuesta sobre la base de la solución del derecho alemán", Revista de información fiscal, $\mathrm{n}^{\mathrm{o}}$ 61, 2004, pp. 27-81. 
- MENÉNDEZ MORENO, A., "De la anécdota a la categoría: (a propósito de las consultas vinculantes y del régimen de estimación objetiva)", Quincena fiscal, $\mathrm{n}^{\circ} 12$, 2014, pp. 11-17.

- PAVÓN PÉREZ, J. A., "El Derecho administrativo a través de la consulta de bases de datos jurídicas accesibles en red y la utilización de redes sociales (blogs jurídicos)", en CAMISÓN YAGÜE, J. A. (Dir.), Experiencias de Innovación docente en los Estudios Jurídicos: una visión práctica [Sevilla]: Grupo de Investigación «Fiscalitas \& Iuris» de la Universidad de Extremadura, 2016, pp. 130-136.

- PÉREZ CARRILLO, E. F., "Bases de datos electrónicas como apoyo a la docencia y a la coordinación de contenidos impartidos en los estudios jurídicos: el ejemplo de Eur Lex", en ECHEBARRÍA SÁENZ, M. (Dir.), Coordinación y planificación en los estudios de Derecho [Recurso electrónico]: 15 al 16 de septiembre 2012: Universidad de Valladolid, Valladolid : Universidad de Valladolid, 2012, pp. 142-171.

- PÉREZ DEL HOYO, R.; ANDÚJAR-MONTOYA, M. D.; SENTANA GADEA, I.; GONZÁLEZ AVILÉS, Á. B.; GARCÍA GONZÁLEZ, E., "Las bases de datos de acceso libre como metodología docente", en Memorias del Programa de Redes-I3CE de calidad, innovación e investigación en docencia universitaria: Convocatoria 2017-18, Alicante: Universidad de Alicante, Instituto de Ciencias de la Educación (ICE), 2018, 2018, pp. 2073-2079.

- VILLAVERDE GÓMEZ, M. B., "Un supuesto de interpretación de las normas tributarias: los efectos jurídicos de las contestaciones a consultas", Dereito: Revista xuridica da Universidade de Santiago de Compostela, $\mathrm{n}^{\mathrm{o}}$ 1, 2013, pp. 685-715. 